\title{
Creep Properties of Cement and Alkali Activated Fly ash Materials using Nanoindentation Technique
}

\author{
Hyuk Lee ${ }^{\mathrm{a}, *}$, Vanissorn Vimonsatit ${ }^{\mathrm{a}}$, Prinya Chindaprasirt ${ }^{\mathrm{b}}$, Tuan $\mathrm{Ngo}^{\mathrm{c}}$, \\ Priyan Mendis ${ }^{\mathrm{c}}$ \\ ${ }^{a}$ Department of Civil Engineering, Curtin University, Western Australia, Australia \\ ${ }^{b}$ Sustainability Infrastructure Development and Research Centre, Department of Civil \\ Engineering, Faculty of Engineering, Khon Kaen University, Thailand \\ ${ }^{c}$ Department of Infrastructure Engineering, University of Melbourne, Victoria, Australia
}

\begin{abstract}
This paper presents creep properties of cement and alkali activated fly ash (AAFA) paste and mortar determined from statistical analysis of nanoindentation data. Cement paste having $95 \mathrm{MPa}$ compressive strength at 28 days was tested for comparison and validation with a conventional test. Using nanoindentation, the specific creep of the cement paste after one year was predicted as 18.32 microstrain/MPa. For AAFA samples, an experimental program was set up using Taguchi's Design of Experiment method to consider four parameters, silica fume, sand to binder ratio, liquid to solid ratio, and superplasticiser, each with three variations. Using ANOVA, the percentage contributions of these parameters on the creep modulus of AAFA samples are: silica fume $26 \%$, sand to binder ratio $21 \%$, liquid to solid ratio $22 \%$, and superplasticiser $31 \%$. The results using deconvolution technique to identify the creep modulus of different phases of AAFA matrices show that partly-activated, non-activated slag and non-activated compact glass phases are leading the creep behaviour of AAFA samples due to their high creep modulus. Compare to other parameters, the liquid to solid ratio contributes the most to the creep property of partly-activated slag, non-activated slag and non-activated compact glass phases, that is, $51 \%$,
\end{abstract}

\footnotetext{
${ }^{*}$ Corresponding author, Tel: +61 433390054

Email addresses: leehyuk1982@gmail.com (Hyuk Lee), V.Vimonsatit@curtin.edu.au (Vanissorn Vimonsatit), prinya@kku.ac.th (Prinya Chindaprasirt), dtngo@unimelb.edu.au (Tuan Ngo), pamendis@unimelb.edu.au (Priyan Mendis)
} 
$89 \%, 68 \%$, respectively. Sand to binder ratio and superplasticiser have minor effect on the creep behaviour. The results of the creep properties of AAFA paste were then compared with those of AAFA concrete using an upscaling process. The creep rate of AAFA concrete was defined by the creep properties of the matrix and the interface between aggregates and matrix assuming perfect bonding and slip bonding conditions. The results from the upscaling process show that the creep properties of AAFA paste from nanoindentation are representative of the long-term creep properties of AAFA concrete determined from a conventional test method.

Keywords: Alkali Activated Cement Geopolymer Fly ash based cement Creep Viscoelasticity Nanoindentation

\section{Introduction}

Durability of structural materials is an important aspect in the designed life of a structure. Concrete, being the most used structural material in construction, is known to have viscoelastic properties. After a long sustained load, strain

5 in concrete increases with time which exhibits creep [1]. Determining creep properties of concrete and factors influencing creep are necessary when aiming for a more sustainable design. Concrete is conventionally made of ordinary Portland cement (OPC), but due to concerns over the environmental impact caused by cement production, alternative low-carbon cementitious materials have been introduced as a full or partial replacement of OPC in producing concrete. These materials are such as blended cement, alkali-activated pozzolan cement, and Geopolymer. This paper presents the creep properties of some of these materials and how different compositions in the mixtures are affecting their creep properties. The main aim is to obtain the optimum mix design, within the range of the parameters considered, for the minimum creep behaviour.

The measurement of viscoelastic properties of a material can be obtained by considering the nature and the rate of configurational rearrangements and the interaction of the properties. Creep testing involves applying a constant instan- 
taneous stress to the specimen and measuring strain as a function of time during stressing [2]. The creep properties of cementitious materials are still an enigma because of the difficulty in linking to the timescale involved. The complex creep behaviour of cementitious material is largely related to the viscoelastic response of the primary hydration or reaction products and the binding phase of hardening [3. However, Fischer-Cripps et al. 4] and Vandamme et al. 3, 5, 6] presented that time-dependent viscoelastic properties such as creep behaviour could be measured by nanoindentation technique.

The nanostructure of concrete made with OPC is controlled by the structure of calcium silicate hydrated (CSH) which governs its fundamental properties, such as strength, relaxation, creep, and fracture behaviour. Determining these propso erties demands a detailed knowledge of the nanostructure and how it relates to local mechanical properties. Thus, it is important to understand the nanostructure of OPC pastes. Several techniques have been used to understand CSH structures. Thomas et al. [7] determined two CSH morphologies using nitrogen, and the content of the two CSH phases depended on varying parameters such as internal water content. Similarly, Tennis et al. 8] provided a microstructure model of OPC paste that could quantitatively predict the volume of various phases. This model provides a mean for quantifying a volumetric proportion of major hydration products such as capillary porosity (MP), low-density CSH (LD CSH), and high-density CSH (HD CSH). Constantinides et al. 9, 10] de40 termined two types of $\mathrm{CSH}$, Portlandite $(\mathrm{CH})$ and clinker with nanoindentation. The results show that decalcification of CSH phases are the primary source of nanometre-scale elastic modulus degradation. Generally, the complex viscous characteristic of OPC pastes is according to the viscous behaviour of CSH, which is the primary hydration products of OPC paste. Advances in cement 45 technology have recently enabled the understanding of the properties of CSH. However, CSH exhibits significant local variations and is still difficult to probe into its overall characteristics directly. Thus, a study on viscoelastic properties of hydration products by nanoindentation is well-suited, making it possible to probe sub-micrometric volume of material [11]. 
Alkali activated cement (AAC) has pozzolan as the main constituent, which can react with alkali to form binder [12]. The composites of cementitious components and alkali activated pozzolan cement are classified according to the type of pozzolan such as fly ash, metakaolin, soda lime glass and natural pozzolan [13. Several researchers reported that common chemical compositions of traditional Portland cement and alkali activated pozzolan cement are silica and alumina which can also be found in industrial by products [1, 14. Though, several macro characteristics of AAC prepared from different aluminosilicate sources may appear similar, their physico-chemical and thermal properties vary to a large extent depending predominantly on the raw materials used [15. Generally, AAC is a source material that does not contain carbonate, therefore, it does not release vast quantities of $\mathrm{CO}_{2}$ as compared to traditional Portland cement. Recently, some research articles presented that AAC has several phases that contribute to the physical properties of the material. Němeček et al. [16] identified the reaction products of alkali activated fly ash cement (AAFA) pastes as N-A-S-H (sodium aluminosilicate hydrate), partly activated slag (N-A-S-H gel intermixed with slag-like particles), non-activated slag (porous non-activated slag-like particles), and non-activated slag (solid non-activated glass sphere or their relicts) using nanoindentation. Similarly, Lee et al. [14 investigated the effect of varying factors on the physical properties of AAFA and also found that 70 N-A-S-H phase was a major reaction product of AAFA.

Viscoelastic properties of a material can be under the influence of time-dependent stress-strain factors. Previously, the method of analysis of nanoindentation assumed that behaviours of material are in an elastic-plastic manner. However, time-dependent viscoelastic properties, such as creep, can occur under indentation impress [17, 18. Literature shows that indentation properties of OPC and AAFA are well known from several research studies, but microporomechanics and creep behaviour of hydration products of OPC are still not well determined. This research is to fill this gap by investigating the creep properties of OPC and AAFA matrices using micromechanics, Creep properties are studied and comso pared with the results from conventional test methods. This study also reveals 
Table 1: Chemical composition of low calcium fly ash (wt.\%)

\begin{tabular}{ccccc}
\hline $\mathrm{SiO}_{2}$ & $\mathrm{Al}_{2} \mathrm{O}_{3}$ & $\mathrm{CaO}$ & $\mathrm{Fe}_{2} \mathrm{O}_{3}$ & $\mathrm{~K}_{2} \mathrm{O}$ \\
\hline 65.9 & 24.0 & 1.59 & 2.87 & 1.44 \\
\hline
\end{tabular}

how different chemical phases in the matrices affect the creep properties, and suggests an optimum mix design to minimise creep.

\section{Methodology}

\subsection{Materials and experimental procedure}

Taguchi's Design of Experiment (DoE) is an efficient method to determine the contribution of parameters on the tested properties within the ranges considered [19]. In this investigation, two series of samples were tested, OPC and AAFA mixtures. OPC properties were tested for comparison and validation with the properties obtained from conventional tests. General purpose (Type

90 I) cement available locally in Australia with the water to cement ratio of 0.3 was used to prepare the OPC paste samples. The samples were cured in lime water at ambient temperature, $23 \pm 3^{\circ} \mathrm{C}$. The average compressive strength of the samples was obtained as $94.6 \mathrm{MPa}$ at 28 days curing age. Nanoindentation test was carried out on the three sets of samples with minimum 300 indentation points per sample.

Nine different AAFA mixtures were prepared according to Taguchis DoE approach. In the authors previous research [14, four variables viz., silica fume $(\mathrm{SF})$, sand to binder ratio $(\mathrm{s} / \mathrm{c})$, liquid to solid ratio $(\mathrm{l} / \mathrm{s})$ and superplasticiser (SP) contents were the parameters considered in the experimental program. The fly ash used was from Collie Power station in Australia, the chemical composition of fly ash is given in Table 1 .

The medium particle size and Loss on Ignition (LOI) of fly ash were $45 \mu \mathrm{m}$ and $1.5 \%$, respectively. The experiment work in this research is to determine creep characteristics of AAFA matrices by considering three levels of the parameters.

The standard $L_{9}\left(3^{4}\right)$ orthogonal array defining the contents of the mixtures is 
as shown in Table 2 and 3 The data obtained from the nanoindentaion test can be used to determine meaningful mechanical properties of the material [18]. The following section will provide an overview of nanoindentation technique and equations necessary for statistical analysis in this research.

\subsection{Principle and microporomechanics}

Measurement of elastic modulus and hardness of material can be obtained from indentation load-displacement data 20,22 . Hertz contact equation determines the indentation modulus $M$ as:

$$
\frac{1}{M}=\frac{1-\nu^{2}}{E}+\frac{\left(1-\nu^{\prime 2}\right)}{E^{\prime}}
$$

where $E$ is the elastic modulus of the specimens and $n u$ is the Poisson's ratio of the indented material, while the superscript prime denotes the corresponding properties of the indenter, that is, $E$ is the elastic modulus and $n u$ is the Poisson's ratio of indenter. The modulus $M$ and hardness $H$ values can then be used to determine the particle properties such as particle stiffness $(\lambda)$, Poisson's ratio $\left(v_{s}\right)$, cohesion $(\kappa)$, friction coefficient $(\zeta)$ and packing density $(\eta)$ as $[14,23$ :

$$
M=\frac{\sqrt{\pi}}{2} \frac{S}{\sqrt{A_{c}}}=\lambda \cdot \Pi_{M}\left(v_{s}, \eta, \eta_{0}\right)
$$

120

$$
\left.H=\frac{P_{\max }}{A_{c}}=\lambda \cdot \Pi_{H}\left(\zeta, \eta, \eta_{(} 0\right)\right)
$$

where $P, S, \Pi_{M}$ and $\Pi_{H}$ are indentation loading, the contact stiffness, dimensional scaling relating to stiffness and hardness, respectively. The term $A_{c}$ is the projected contact area which can be expressed in term of $h_{r}$ and $h_{c}$ as determined by Oliver-Pharr method [20]. The solid percolation threshold $\eta_{0}$ is

Table 2: Parameters and Levels

\begin{tabular}{llccc}
\hline & Parameter & Level 1 & Level 2 & Level 3 \\
\hline SF $(\%)$ & Silica fume & 0 & 2 & 4 \\
\hline s/c & Sand to cementitious material ratio & 0 & 0.25 & 0.5 \\
\hline l/s & Liquid to solid ratio & 0.6 & 0.65 & 0.7 \\
\hline SP $(\%)$ & Superplasticiser & 0 & 2 & 4 \\
\hline
\end{tabular}


Table 3: Mix proportion

\begin{tabular}{cccccc}
\hline Mix No. & Fly ash & SF & s/c & l/s & SP \\
\hline 1 & 1 & 0 & 0 & 0.6 & 0 \\
2 & 1 & 0 & 0.25 & 0.65 & 0.02 \\
3 & 1 & 0 & 0.5 & 0.70 & 0.04 \\
4 & 1 & 0.02 & 0 & 0.65 & 0.04 \\
5 & 1 & 0.02 & 0.25 & 0.70 & 0 \\
6 & 1 & 0.02 & 0.5 & 0.60 & 0.02 \\
7 & 1 & 0.04 & 0 & 0.70 & 0.02 \\
8 & 1 & 0.04 & 0.25 & 0.60 & 0.04 \\
9 & 1 & 0.04 & 0.5 & 0.65 & 0
\end{tabular}

125 linear micromechanics [14, 23:

$$
\Pi_{H}(\zeta)=\Pi_{0} \times\left[1+(1-\eta) \zeta-(d-e \cdot \eta) \zeta^{2}-(f-g \cdot \eta) \zeta^{5}\right]
$$

in which a function of frictionless portions $\Pi_{o}$ is:

$$
\Pi_{0}=\frac{12 \eta(a-b \cdot \eta)[(2 \eta-1)(2+\eta)]^{0.5}}{(1-c \cdot \eta)(2+\eta)}
$$

Based on micromechanics, the coefficients suitable for Berkovich indenter are: $a=0.19567, b=0.03739, c=0.77999, d=20.3138, e=31.5352, f=52.1817$ 135 and $g=99.3465$ with $\eta=0.5$ [23. Equation (2) and (3) can be treated as 
a quadratic minimisation problem between the experimental values and the theoretical scaling relationship by an inverse application that solid properties and local packing density are unknowns [24, 25].The problem can be written as:

$$
\min \sum_{i=1}^{N}\left[\left(M_{i}-\lambda \cdot \Pi_{M}\left(v_{s}, \eta_{i}\right)\right)-\left(H_{i}-\kappa \cdot \Pi_{H}\left(\zeta, \eta_{i}\right)\right)\right]^{0.5}
$$

where $N$ is the total number of indentation points; $M_{i}$ and $H_{i}$ are determined from Equations (2) and (3), respectively. Solving Equation (8) will deliver the analytical results of the scaling relationship between $M, H$ and $\eta$.

\subsection{Deconvolution technique}

A statistical technique is required for the analysis of heterogeneous or composite materials which are normally complex. The deconvolution technique starts with the generation of experimental cumulative distribution function $(\mathrm{CDF})$. It is more convenient to deconvolute CDF rather than the probability density function (PDF) because CDF does not require a choice of bin size for histogram construction [14, 23]. Therefore, the experimental CDF can be written as:

$$
\Phi^{e}(X)=\frac{i}{N}-\frac{1}{2 N} \quad \text { for } i \in[1, N]
$$

where $X$ is a type of the mechanical properties, and $N$ is the number of indentation tests. Gaussian distribution gives the theoretical CDF for each phase as:

$$
\Phi_{j}^{t}(X)=\frac{1}{\sigma_{j} \sqrt{2 \pi}} \int_{\infty}^{X_{i}} \exp \left(\frac{-\left(s-\mu_{j}\right)^{2}}{2 \sigma_{j}^{2}}\right) d t \quad \text { for } i \in[1, N]
$$

where $\mu_{j}$ is the mean, $\sigma_{j}$ is the standard deviation and $j$ is a phase. The theoretical CDF function can be obtained by minimising the error function as:

$$
\min \sum_{i=1}^{N} \sum\left(f_{j} \cdot \Phi_{j}^{t}(X)-\Phi^{e}(X)\right)^{2}
$$

155

Moreover, the total volume fraction of the material phases can be expressed by summation of total single phase $f_{j}$ as:

$$
\sum_{j=1}^{n} f_{j}=1
$$


where $n$ is the total number of phases. In addition, the constraint of the minimisation problem is required to avoid the overlapping of each phase as:

$$
\mu_{j}(X)+\sigma_{j}(X) \leq \mu_{j+1}(X)+\sigma_{j+1}(X)
$$

Therefore, the mechanical properties and surface volume fraction of each phase can be determined from the mean and standard deviation of the deconvolution technique.

\subsection{Creep properties}

According to Vandamme et al. [5, 6, nanoindentation can be used to determine the quantitative viscoelastic properties of material. Also, they studied geometric relationship between indenter and contact problem during indentation. As viscoelastic behaviour is the role played by time, if the material is ideally viscos fluid, the stress can be instantaneously infinite under constant strain. However, actual material behaviour shows that under constant strain, the stress generally decreases from its initial value rapidly and later more gradually. This behaviour is known as stress relaxation. Another important behaviour is creep, i.e., the deformation of viscoelastic materials under constant stress increases with time. The creep compliance rate $\dot{J}_{c}(t)$ can be expressed [5, 6] as:

$$
\dot{J}_{c}(t)=\frac{2 a(t)}{P_{\max }} \dot{h}(t)
$$

where $a(t)$ is the radius of the projected contact area, $P_{\max }$ is maximum indentation load, and $\dot{h}(t)$ is the change in the indentation depth over the holding time, which can be expressed by a single power logarithm function as:

$$
\dot{h}(t)=x_{1} \ln \left(\frac{t}{x_{2}}+1\right)+x_{3} t+x_{4}
$$

where $x_{1}, \ldots, x_{4}$ are constants. In Equation (14), the material related term is only the logarithmic term [5, 6, thus, $\dot{h}(t)$ can be replaced by the term of $x_{1} / t$. From the Equation (14), the long-term contact creep compliance rate is given by:

$$
\dot{J}_{c}(t)=\frac{1}{C t}, \quad \text { where } \quad C=\frac{P_{\max }}{2 a_{c} x_{1}}
$$


where $C$ is the contact creep modulus, and ac is the contact radius of the maximum projected area between the indenter and the indented sample just before the unloading, i.e., $a_{c} \approx \sqrt{A_{c} / \pi}$. Thus, a higher contact creep modulus will lead to a lower logarithm creep of the material. The equations provided in this section will be used for performing statistical analysis of indented data.

185 The analyses included in this study are microporomechanics, deconvolution, and creep properties.

\section{Result and Discussion}

\subsection{Indentation creep properties of $O P C$}

The results of deconvolution to determine the indentation modulus, $M$, and hardness, $H$, are illustrated in Figure 1 and given in Table 4 . The mean values of the first peak shows $M=9.388 \mathrm{GPa}, H=0.297 \mathrm{GPa}$; the second peak, $M=16.591 \mathrm{GPa}, H=0.700 \mathrm{GPa}$; the third peak, $M=29.818 \mathrm{GPa}$, $H=1.412 \mathrm{GPa}$, which are in good agreement with the values reported in literatures [23, 26]. For the fourth peak, $M=48.677 \mathrm{GPa}, H=10.495 \mathrm{GPa}$, and the clinker, $M=112.378 \mathrm{GPa}, H=14.718 \mathrm{GPa}$.

Pipilikaki et al. 27] reported that binding cementitious material is porous with small pore sizes. Thus, it can be assumed that the characteristic size of the porosity is much smaller than the maximum indentation depth $(h)$ for scaling separability reason [23. With the hypothesis that OPC paste is a porous ma-

Table 4: Deconvolution results of indentation modulus and hardness of OPC paste

\begin{tabular}{|c|c|c|c|c|c|}
\hline \multirow[t]{2}{*}{ Phase } & \multicolumn{2}{|c|}{$\begin{array}{c}\text { Modulus } \\
(\mathrm{MPa})\end{array}$} & \multicolumn{2}{|c|}{$\begin{array}{c}\text { Hardness } \\
(\mathrm{MPa})\end{array}$} & \multirow{2}{*}{$\begin{array}{c}\text { Volume } \\
\text { fraction } \\
\%\end{array}$} \\
\hline & mean & StD & mean & Std & \\
\hline MP & 9.388 & 3.562 & 0.297 & 0.016 & 43 \\
\hline LD-CSH & 16.591 & 4.712 & 0.700 & 0.147 & 34 \\
\hline HD-CSH & 29.818 & 3.984 & 1.412 & 0.215 & 10 \\
\hline $\mathrm{CH}$ & 48.677 & 10.495 & 2.352 & 1.239 & 8 \\
\hline Clinker & 112.378 & 29.557 & 14.718 & 8.027 & 5 \\
\hline
\end{tabular}



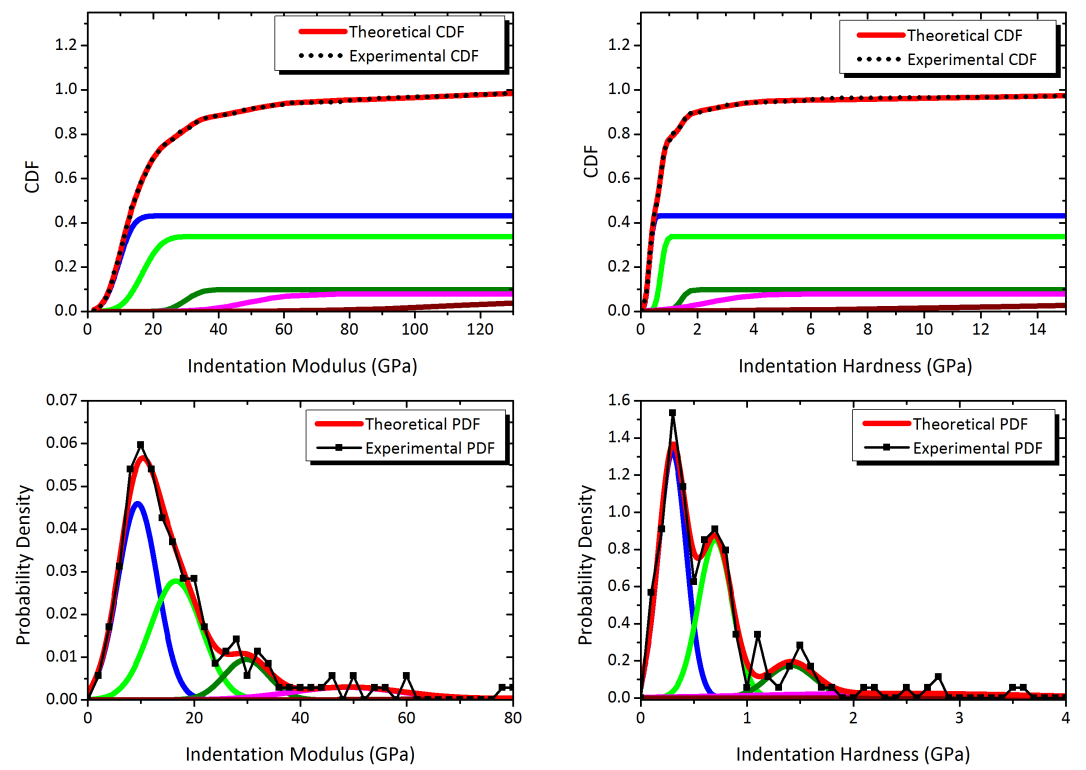

Figure 1: Deconvolution results of indentation modulus and hardness of OPC paste

LD CSH phase has a characteristic $\eta=0.556 \pm 0.01$ with $33 \%$ of volume fraction; $\mathrm{HD} \mathrm{CSH}$ phase has $\eta=0.595 \pm 0.01$ with $10 \%$ of volume fraction; $\mathrm{CH}$ phase 

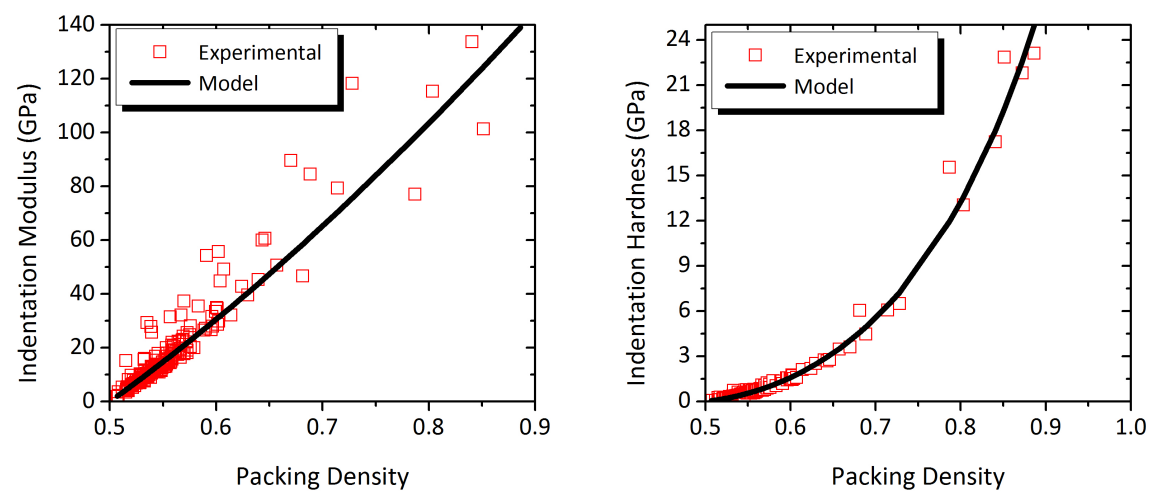

Figure 2: Packing Density $(\eta)$ distribution of OPC paste
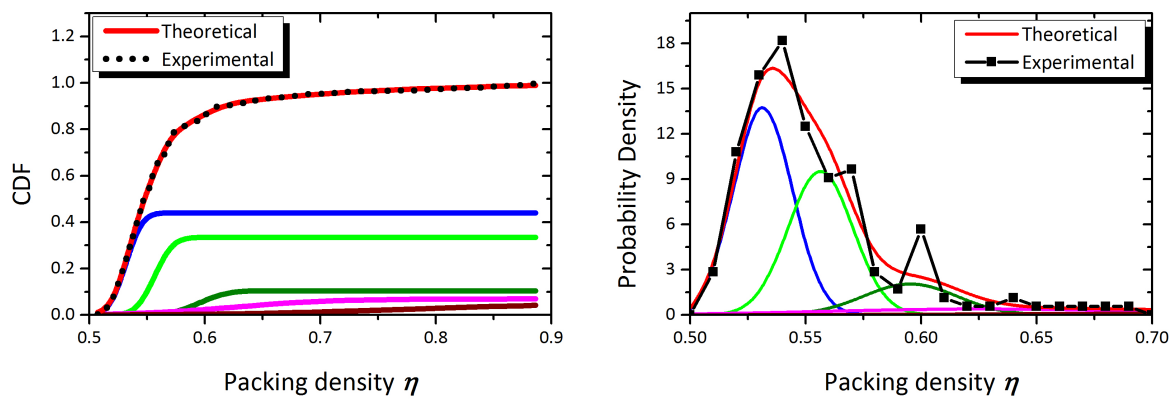

Figure 3: Deconvolution results of packing density $(\eta)$ of OPC paste

Table 5: Packing density $(\eta)$ of OPC paste

\begin{tabular}{llc}
\hline \multirow{2}{*}{ Phase } & \multicolumn{2}{c}{ Packing Density } \\
\cline { 2 - 3 } & mean & StD \\
\hline MP & 0.531 & 0.01 \\
\hline LD-CSH & 0.556 & 0.01 \\
\hline HD-CSH & 0.595 & 0.01 \\
\hline CH & 0.627 & 0.06 \\
\hline Clinker & 0.780 & 0.12 \\
\hline
\end{tabular}

has $\eta=0.627 \pm 0.06$ with $7 \%$ of volume fraction; clinker has $\eta=0.780 \pm 0.12$ with $5 \%$ of volume fraction.

In order to determine the creep behaviour using nanoindentation test, the change 
in the depth of the holding phase can be fitted with a logarithmic function as presented in Equation (15). The curve fitting was performed by MATLAB 28] and the average corrected coefficient of the fitted curve was observed as $0.986 \pm 0.04$. The typical curve fitting of the penetration depth versus time history is presented in Figure 4 With the logarithm curve fitting results, $x_{1}$, the long-term contact creep compliance rate was obtained as 409.266 GPa. The result of the creep compliance rate clearly shows that the rate of the creep compliance sharply decreases after a few days of stressing. In addition, the deconvolution technique was applied to identify the contact creep modulus $C$ of each phase. The greater $C$ value represents the lower rate of the creep. Table 6 summarises the results of $C$ with deconvolution input parameters. For MP phase, $C=75.552 \pm 37.754 \mathrm{GPa}$; LD CSH phase, $C=162.201 \pm 37.754 \mathrm{GPa}$; HD-CSH phase, $C=333.797 \pm 46.979 \mathrm{GPa}$; and $\mathrm{CH}$ phase, $C=663.387 \pm 188.444 \mathrm{GPa}$.

${ }_{230}$ As can be seen from the results, MP phase has the highest creep compliance in OPC pastes which leads to a lower creep modulus. This means that when the porosity increases, it tends to increase the creep compliance. The tested OPC paste had the compressive strength of $95 \mathrm{MPa}$ at 28 days. The specific creep after one year from nanoindentation testing was predicted as $18.32 \mathrm{mi}$ crostrain/MPa. This result agrees well with the literature which indicates that the creep of OPC after one year is to be around 20 microstrain/MPa with respect to the compressive strength at 28 days curing age of concrete [29]. Thus, nanoindentation technique can identify the creep behaviour of a material from a short period indentation test.

\subsection{Indentation creep properties of AAFA}

After conducting indentation creep experiments of AAFA mixtures, creep properties were analysed according to the procedure described in previous section. Figure 5 presents the results of the creep compliance rates of all the nine mixtures. The average corrected coefficient and coefficient of logarithmic curve fitting obtained are as shown in Table 7 . The results of the curve fitting show that the corrected coefficient (R-square) was over 0.98 (98\%). The results of the 


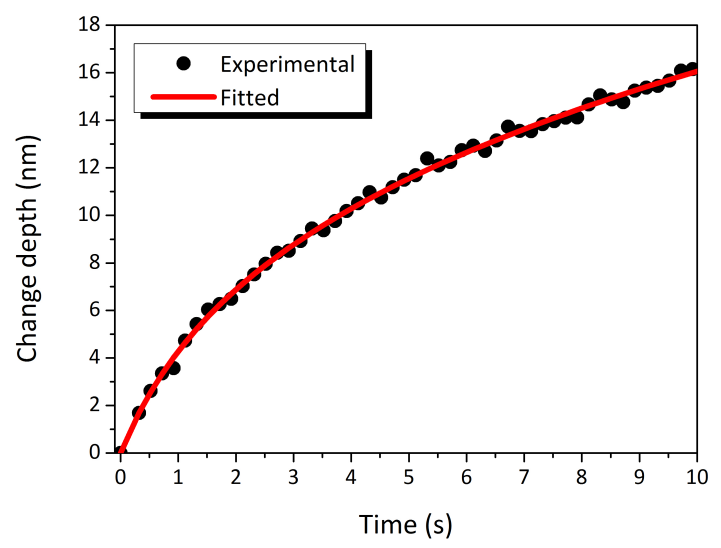

Figure 4: Typical logarithm curve fitting in creep phase

Table 6: Contact creep modulus $(C)$ of OPC paste

\begin{tabular}{lcr}
\hline \multirow{2}{*}{ Phase } & \multicolumn{2}{c}{ Contact creep modulus (MPa) } \\
\cline { 2 - 3 } & mean & \multicolumn{1}{c}{ StD } \\
\hline MP & 75.552 & 37.754 \\
\hline LD-CSH & 162.201 & 53.582 \\
\hline HD-CSH & 333.797 & 46.979 \\
\hline CH & 663.987 & 184.444 \\
\hline Clinker & 3948.108 & 1388.759 \\
\hline
\end{tabular}

contact creep modulus show that Mix 2 and Mix 4 had the lowest and highest contact creep modulus of $336.803 \mathrm{GPa}$ and $2172.179 \mathrm{GPa}$, respectively. In order to understand the meaning of the creep behaviour of AAFA, statistical analysis results were presented to demonstrate the influence of the creep behaviour with varying parameters. The results of the signal to noise $(\mathrm{S} / \mathrm{N})$ ratio show that an increase in the s/c ratio leads to an increase in the creep compliance rate due to a decrease in the creep modulus. The percentage contribution of the four parameters on the creep modulus property from ANOVA are: SF $26.15 \%$, s/c ratio $20.65 \%, 1 / \mathrm{s}$ ratio $22.37 \%$, and SP $30.83 \%$, as shown in Table 8 . ANOVA results provide important information that the creep behaviour of AAFA was influenced by not only a specific parameter but also several parameters in the 
Table 7: Average logarithmic coefficient and corrected coefficient

\begin{tabular}{ccccccc}
\hline \multirow{2}{*}{ Mix No. } & $x_{1}$ & $x_{2}$ & \multicolumn{1}{c}{$x_{3}$} & $x_{4}$ & \multicolumn{1}{c}{$C$} & R-square \\
\cline { 2 - 6 } & $(\mathrm{nm})$ & $(\mathrm{s})$ & $(\mathrm{nm} / \mathrm{s})$ & $(\mathrm{nm})$ & $(\mathrm{GPa})$ & \\
\hline 1 & 11.935 & 3.037 & -0.005 & 0.136 & 386.756 & 0.986 \\
\hline 2 & 10.395 & 3.123 & 0.015 & 0.151 & 336.803 & 0.988 \\
\hline 3 & 17.626 & 4.437 & 0.035 & 0.145 & 504.181 & 0.981 \\
\hline 4 & 7.577 & 14.485 & -0.058 & 0.151 & 2172.179 & 0.982 \\
\hline 5 & 9.159 & 71.840 & -0.089 & 0.139 & 536.120 & 0.983 \\
\hline 6 & 8.236 & 3.412 & -0.074 & 0.151 & 377.457 & 0.982 \\
\hline 7 & 6.365 & 2.160 & -0.051 & 0.135 & 397.848 & 0.987 \\
\hline 8 & 14.230 & 2.508 & 0.117 & 0.152 & 503.422 & 0.987 \\
\hline 9 & 8.057 & 2.497 & -0.051 & 0.113 & 486.692 & 0.984 \\
\hline
\end{tabular}

Table 8: ANOVA results on creep modulus

\begin{tabular}{rrrrrr}
\hline & Source & $\mathrm{DF}^{a}$ & $\mathrm{SS}^{b}$ & $\mathrm{MS}^{c}$ & Contribution \% \\
\hline \multirow{3}{*}{ Creep modulus } & $\mathrm{SF}$ & 2 & 706711 & 353356 & 26.15 \\
\cline { 2 - 6 } & $\mathrm{S} / \mathrm{c}$ & 2 & 557892 & 278946 & 20.65 \\
\cline { 2 - 6 } & $\mathrm{l} / \mathrm{s}$ & 2 & 604564 & 302282 & 22.37 \\
\cline { 2 - 6 } & $\mathrm{SP}$ & 2 & 833046 & 416523 & 30.83 \\
\hline \multicolumn{4}{c}{${ }^{a}$ degree of freedom ${ }^{b}$ sum of square ${ }^{c}$ mean square }
\end{tabular}

\section{AAFA matrix.}

In addition, the deconvolution technique was used to identify the creep modulus $\mathrm{C}$ of each phase in the matrix as shown in Table 8. The deconvolution results of the creep modulus show that N-A-S-H is mainly the phase that increases the creep compliance of AAFA due to smaller creep modulus. Partly-activated and non-activated phases in which the silicon and aluminium composition are not completely dissolved in the alkaline solution (block-polymerisation) show smaller creep behaviour because of having higher creep modulus than that of the N-A-S-H phase from Equation 14. Therefore, partly-activated and nonactivated phases are leading the creep behaviour of AAFA due to high creep modulus. The polymerisation takes place only on the surface of the atom and is adequate to form the blocks to produce the polymer binder in AAFA [30. 

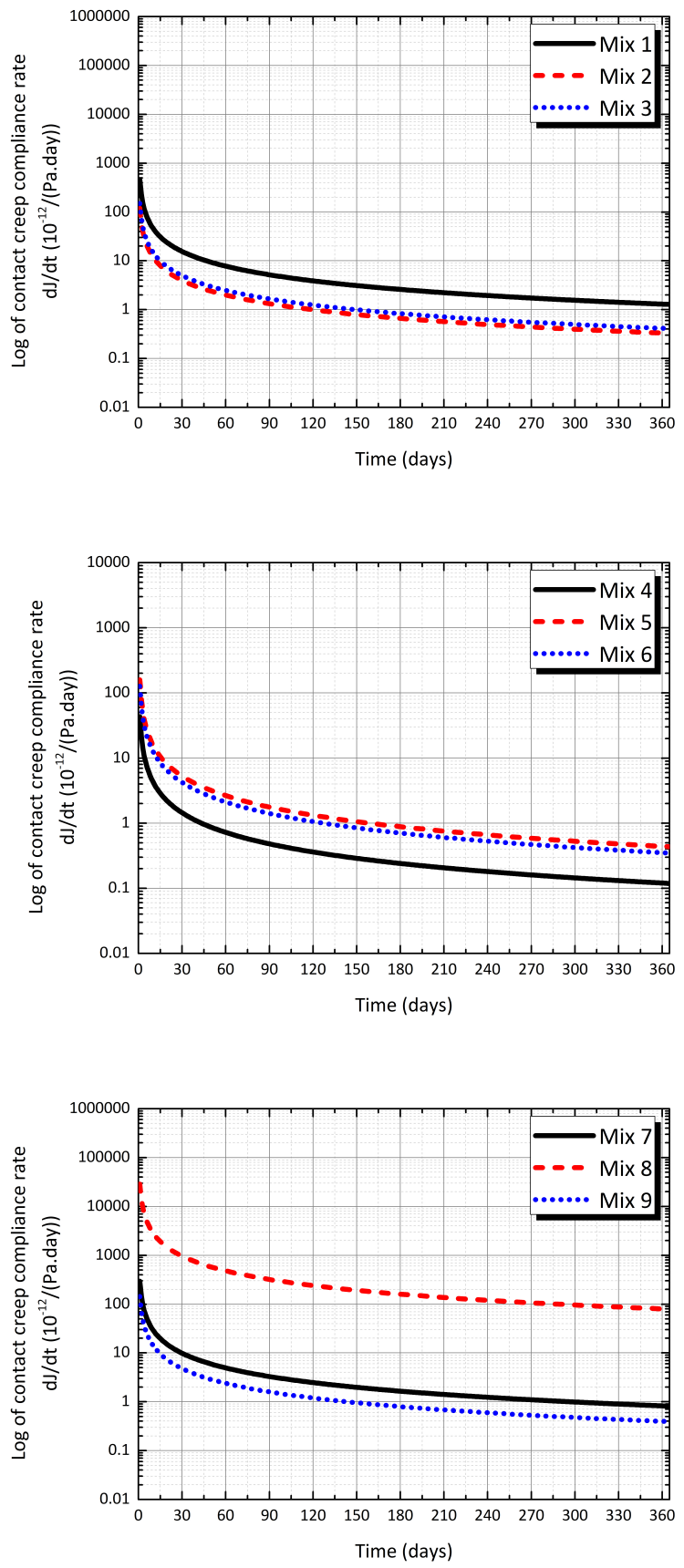

Figure 5: Contact creep compliance rate of AAFA 
The $\mathrm{S} / \mathrm{N}$ ratio was analysed to understand the effect of parameters on the creep modulus of partly-activated and non-activated phases. As presented in Figure 6. $\mathrm{l} / \mathrm{s}$ ratio is the most significant parameter on the creep modulus of partly and non-activated phases. An increase in $1 / \mathrm{s}$ ratio leads to a decrease in the creep modulus, thus, an increase in $1 / \mathrm{s}$ ratio would be a cause of increasing the creep

275 behaviour of AAFA. Similarly, ANOVA was performed on the results of the creep modulus of partly-activated and non-activated phases as shown in Table 10. The results show that the contribution of $1 / \mathrm{s}$ ratio on the creep modulus of partly-activated slag, non-activated slag, and non-activated compact glass are $50.74 \%, 88.69 \%$ and $68.08 \%$, respectively, while the s/c ratio and SP have minor effect on the creep behaviour of AAFA. In addition, the $\mathrm{S} / \mathrm{N}$ ratio shows that an increase of SF up to $2 \%$ leads to an increase in the creep modulus of partly-activated slag, non-activated slag, and non-activated compact glass. It is noted that increasing the SF up to $2 \%$ of partly activated slag has $40.47 \%$ of contribution, thus increasing SF up to $2 \%$ would cause creep modulus reduction. In turn, this increase of SF up to $2 \%$ entails a decrease in N-A-S-H phase. According to Lee et al. 14 the SF available for reaction decrease the amount of N-A-S-H, it also decrease the N-A-S-H porosity. The decrease in the amount $\mathrm{N}-\mathrm{A}-\mathrm{S}-\mathrm{H}$ phase favours the formation of denser packed N-A-S-H phase.

\subsection{Comparison}

In this section the obtained creep modulus of AAFA paste mixture (Mix 1) is compared with the value obtained from the experiment of AAFA concrete by Wallah et al. 30. The tested AAFA concrete was made of $77 \%$ of aggregates (in volume of concrete) and the liquid to solid ratio was 0.35 . For a large time, $t$, Wallah et al. 30] suggested that the creep compliance $J(t) \approx C^{u n i} \cdot \ln (t)$, where $J(t)$ after one year was $15 \times 10^{-6} / \mathrm{MPa}$, and the uniaxial creep modulus $C^{\text {uni }}=868.727 \mathrm{GPa}$. In the present case, AAFA mixtures had $\mathrm{l} / \mathrm{s}=0.6$, and from ANOVA, the percentage contribution of the $1 / \mathrm{s}$ ratio on the creep modulus is $22.37 \%$, as presented in Table 7 . Thus, the creep modulus can be calculated as $C^{\text {uni }}=868.727 \times(0.35 / 0.6 \times 22.37 \%)=113.36 \mathrm{GPa}$. Noting that the $C^{\text {uni }}$ 


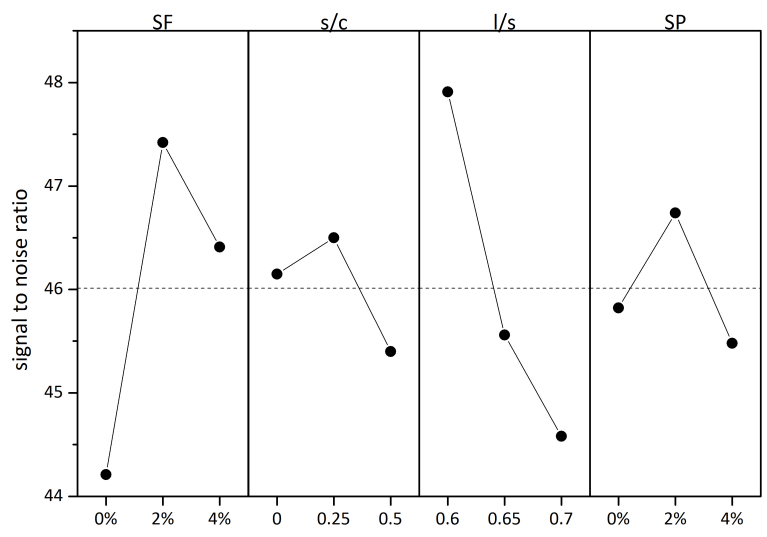

(a) Partly-activated slag (larger is better)
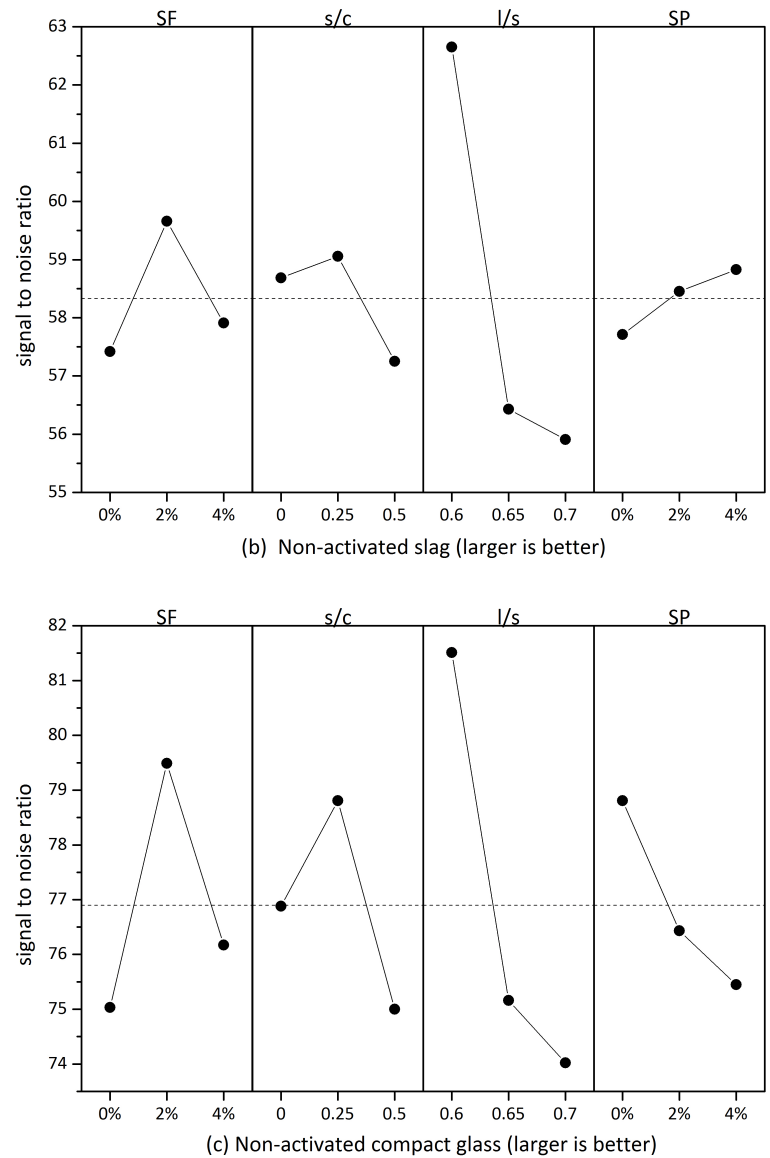

Figure 6: Effect of parameters on creep modulus of partly-activated and non-activated phases 
Table 9: Deconvolution results of Creep Modulus $(C)$ (Unit in GPa)

\begin{tabular}{rrrrrrrrr}
\hline \multirow{2}{*}{ Mix } & \multicolumn{2}{c}{ A } & \multicolumn{2}{c}{ B } & \multicolumn{2}{c}{ C } & D \\
\cline { 2 - 9 } & mean & StD & mean & StD & mean & StD & mean & StD \\
\hline 1 & 61.597 & 38.354 & 200.611 & 78.705 & 1185.385 & 742.521 & 11946.131 & 5933.313 \\
\hline 2 & 78.028 & 35.566 & 177.069 & 80.719 & 658.149 & 403.671 & 5451.601 & 3687.509 \\
\hline 3 & 40.982 & 29.609 & 120.580 & 60.085 & 526.086 & 270.479 & 2757.519 & 1485.664 \\
\hline 4 & 63.828 & 28.705 & 212.993 & 89.196 & 853.733 & 508.367 & 6518.359 & 4303.578 \\
\hline 5 & 65.646 & 35.395 & 206.029 & 61.857 & 737.200 & 396.137 & 10523.844 & 6704.933 \\
\hline 6 & 98.314 & 60.987 & 296.108 & 119.977 & 1415.070 & 704.133 & 12209.248 & 6868.191 \\
\hline 7 & 92.288 & 37.273 & 195.745 & 53.218 & 628.075 & 303.435 & 4371.304 & 3105.210 \\
\hline 8 & 105.965 & 67.850 & 258.409 & 169.916 & 1488.145 & 830.584 & 11545.101 & 6116.926 \\
\hline 9 & 65.515 & 23.356 & 180.902 & 60.882 & 519.336 & 349.451 & 5281.275 & 4111.733 \\
\hline
\end{tabular}

A: N-A-S-H; B:Partly activated slag; C: Non-activated slag; D: Non-activated compact glass

Table 10: ANOVA results on creep modulus of partly-activated and non-activated phases

\begin{tabular}{|c|c|c|c|c|c|}
\hline & Source & $\mathrm{DF}^{a}$ & $\mathrm{SS}^{b}$ & $\mathrm{MS}^{c}$ & Contribution $\%$ \\
\hline \multirow{4}{*}{ Partly-activated slag } & $\mathrm{SF}$ & 2 & 8017.5 & 4008.8 & 40.47 \\
\hline & $\mathrm{s} / \mathrm{c}$ & 2 & 344.6 & 172.3 & 1.74 \\
\hline & $1 / \mathrm{s}$ & 2 & 10051.4 & 5025.7 & 50.74 \\
\hline & $\mathrm{SP}$ & 2 & 1395.8 & 697.9 & 7.05 \\
\hline \multirow{4}{*}{ Non-activated slag } & $\mathrm{SF}$ & 2 & 68104 & 34052 & 5.99 \\
\hline & $\mathrm{s} / \mathrm{c}$ & 2 & 29827 & 14913 & 2.62 \\
\hline & $1 / \mathrm{s}$ & 2 & 1008916 & 504458 & 88.69 \\
\hline & SP & 2 & 30730 & 15365 & 2.70 \\
\hline \multirow{4}{*}{ Non-activated compact glass } & $\mathrm{SF}$ & 2 & 16521193 & 8260597 & 15.19 \\
\hline & $\mathrm{s} / \mathrm{c}$ & 2 & 9059186 & 4529593 & 8.33 \\
\hline & $1 / \mathrm{s}$ & 2 & 74028816 & 37014408 & 68.08 \\
\hline & $\mathrm{SP}$ & 2 & 9133741 & 4566871 & 8.40 \\
\hline
\end{tabular}

value is the uniaxial creep modulus of the tested AAFA concrete, not of the paste. The next step is therefore to correlate that with the paste properties.

An upscaling technique was introduced by Vandamme et al. 11 that the homogenised creep properties at the scale of cement pastes are considered with a self-consistent homogenisation scheme which is well-adapted for the assumed 

fraction of aggregates $f^{a g g}=0.77, C^{c-b o n d}$ and $C^{c-s l i p}$ are $613.76 \mathrm{GPa}$ and 138.39 GPa, respectively. Thus, $C^{c-s l i p}$ governs the creep property and the obtained value is in the same order as the creep modulus calculated from the experimental value from Wallah et al. [30, that is, $C^{u n i}=113.36 \mathrm{GPa}$ after one 325 year creep experiment. This result indicates that the AAFA concrete creeps with respect to time can be defined by the creep properties of the matrix and interface between aggregates and matrix with different bonding conditions. The results from the upscaling process show that the analytical approach for determining the creep properties of AAFA from nanoindentation can predict the creep properties 

determined from a conventional test method.

\section{Conclusion}

Nanoindentation technology coupled with a statistical analysis and a deconvolution technique are successfully applied to investigate the creep behaviour of cement (OPC) and fly ash AAFA. Based on the results obtained in this study, the following conclusion can be drawn:

- In OPC paste, five main phases, viz., capillary porosity, low density calcium silicate hydrated, high density calcium silicate hydrated, calcium hydroxide and clinker phases, are quantified. The obtained creep compliance rate of the OPC paste shows that the capillary porosity phase is the main phase that increases the creep compliance rate of the OPC matrix. This result shows that the porosity in the matrix leads to more creep.

- In AAFA mixtures, four main reaction products, sodium aluminosilicate hydrate, partly activated slag, non-activated slag and non- activated compact glass phases are quantified. The statistical analysis results provide important information that the creep behaviour of AAFA is affected by several parameters of the AAFA matrix. The creep behaviour reveals that partly-activated and non-activated phases are the main factors for the creep.It was also found that the liquid to solid ratio is the most affecting parameter on creep, that is, an increase in the liquid to solid ratio leads to more creep. The sand to cementitious ratio and superplasticiser have a minor effect on the creep behaviour of AAFA.

- Within the range of the parameters considered in the AAFA mixtures, the optimum mix proportion to minimise the creep behaviour of AAFA is to have $2 \%$ silica fume, sand to cementitious ratio of 0.25 , liquid to solid ratio of 0.6 , and $0 \%$ superplasticiser of mass content. 
- The presented upscaling approach of nanoindentation is able to link the creep properties of AAFA paste to that of AAFA concrete which are comparable with the long-term creep of AAFA concrete determined from a conventional test method.

Nanoindentation is a reliable technique for determining creep properties of multi- phase materials that requires only a small scale and short term testing. It enables quantifying the effect of each material phase on creep, thus, will be useful for developing a mix design to have a better control of the creep behaviour of composite materials.

\section{Acknowledgements}

The authors would like to acknowledge the assistance by Dr.Kornkanok Boonserm, Department of Applied Chemistry, Rajamangala University of Technology in Thailand. The third author would like to acknowledge the support of Khon

370 Kaen University and Thailand Research Fund (TRF) under the TRD Senior Research Scholar Contract No.RTA5780004.

\section{References}

[1] A. M. Neville, Properties of concrete, Vol. 4, Longman London, 1995.

[2] W. N. Findley, F. A. Davis, Creep and relaxation of nonlinear viscoelastic materials, Courier Corporation, 2013. doi:10.1115/1.3424077.

[3] M. Vandamme, F.-J. Ulm, Nanogranular origin of concrete creep, Proceedings of the National Academy of Sciences 106 (26) (2009) 10552-10557. doi:10.1073/pnas.0901033106.

[4] A. Fischer-Cripps, A simple phenomenological approach to nanoindentation creep, Materials Science and Engineering: A 385 (1) (2004) 74-82. 
[5] M. Vandamme, F.-J. Ulm, Viscoelastic solutions for conical indentation, International Journal of solids and structures 43 (10) (2006) 3142-3165. doi:10.1016/j.ijsolstr.2005.05.043.

[6] M. Vandamme, C. A. Tweedie, G. Constantinides, F.-J. Ulm, K. J. Van Vliet, Quantifying plasticity-independent creep compliance and relaxation of viscoelastoplastic materials under contact loading, Journal of Materials Research 27 (01) (2012) 302-312. doi:10.1557/jmr.2011.302.

[7] J. J. Thomas, H. M. Jennings, A. J. Allen, The surface area of cement paste as measured by neutron scattering: evidence for two csh morphologies, Cement and Concrete Research 28 (6) (1998) 897-905. doi: 10.1016/S0008-8846(98)00049-0.

[8] P. D. Tennis, H. M. Jennings, A model for two types of calcium silicate hydrate in the microstructure of portland cement pastes, Cement and

1. Concrete Research 30 (6) (2000) 855-863. doi:10.1016/S0008-8846(00) 00257-X.

[9] G. Constantinides, F.-J. Ulm, The effect of two types of csh on the elasticity of cement-based materials: Results from nanoindentation and micromechanical modeling, Cement and concrete research 34 (1) (2004) 6780. doi:10.1016/S0008-8846(03)00230-8.

[10] G. Constantinides, F.-J. Ulm, The nanogranular nature of $\mathrm{c}^{-\mathrm{s}-\mathrm{h}}$, Journal of the Mechanics and Physics of Solids 55 (1) (2007) 64-90. doi:10.1016/ j.jmps.2006.06.003

[11] M. Vandamme, F.-J. Ulm, Nanoindentation investigation of creep properties of calcium silicate hydrates, Cement and Concrete Research 52 (2013) 38-52. doi:10.1016/j.cemconres.2013.05.006.

[12] C. Shi, A. F. Jiménez, A. Palomo, New cements for the 21st century: the pursuit of an alternative to portland cement, Cement and concrete research 41 (7) (2011) 750-763. doi:10.1016/j.cemconres.2011.03.016. 
[13] C. Shi, D. Roy, P. Krivenko, Alkali-activated cements and concretes, CRC press, 2006. doi:10.4324/9780203390672.

[14] H. Lee, V. Vimonsatit, P. Chindaprasirt, Mechanical and micromechanical properties of alkali activated fly-ash cement based on nano-indentation, Construction and Building Materials 107 (2016) 95-102. doi:10.1016/j. conbuildmat.2015.12.013.

[15] P. Duxson, A. Fernández-Jiménez, J. L. Provis, G. C. Lukey, A. Palomo, J. Van Deventer, Geopolymer technology: the current state of the art, Journal of Materials Science 42 (9) (2007) 2917-2933. doi:10.1007/ s10853-006-0637-z.

[16] J. Němeček, V. Šmilauer, L. Kopeckỳ, Nanoindentation characteristics of alkali-activated aluminosilicate materials, Cement and Concrete Composites 33 (2) (2011) 163-170. doi:10.1016/j.cemconcomp.2010.10.005.

[17] A. C. Fischer-Cripps, Introduction to contact mechanics, Springer, 2000. doi:10.1007/978-0-387-68188-7.

[18] A. C. Fischer-Cripps, Nanoindentation, Springer, 2011. doi:10.1007/ 978-1-4419-9872-9.

[19] R. K. Roy, A primer on the Taguchi method, Society of Manufacturing Engineers, 2010.

[20] W. C. Oliver, G. M. Pharr, An improved technique for determining hardness and elastic modulus using load and displacement sensing indentation experiments, Journal of materials research 7 (06) (1992) 1564-1583. doi:10.1557/JMR.1992.1564.

[21] Y.-T. Cheng, C.-M. Cheng, Scaling, dimensional analysis, and indentation measurements, Materials Science and Engineering: R: Reports 44 (4) (2004) 91-149. doi:10.1016/j.mser.2004.05.001 
[22] G. Constantinides, K. R. Chandran, F.-J. Ulm, K. Van Vliet, Grid indentation analysis of composite microstructure and mechanics: Principles and validation, Materials Science and Engineering: A 430 (1) (2006) 189-202. doi:10.1016/j.msea.2006.05.125

[23] F.-J. Ulm, M. Vandamme, C. Bobko, J. Alberto Ortega, K. Tai, C. Ortiz, Statistical indentation techniques for hydrated nanocomposites: concrete, bone, and shale, Journal of the American Ceramic Society 90 (9) (2007) 2677-2692. doi:10.1111/j.1551-2916.2007.02012.x.

[24] C. Bobko, F.-J. Ulm, The nano-mechanical morphology of shale, Mechanics

[25] C. P. Bobko, B. Gathier, J. A. Ortega, F.-J. Ulm, L. Borges, Y. N. Abousleiman, The nanogranular origin of friction and cohesion in shale a strength homogenization approach to interpretation of nanoindentation results, International Journal for Numerical and Analytical Methods in

[27] P. Pipilikaki, M. Beazi-Katsioti, The assessment of porosity and pore size distribution of limestone portland cement pastes, Construction and Building Materials 23 (5) (2009) 1966-1970. doi:10.1016/j.conbuildmat. 2008.08 .028

[28] Matlab and statistics toolbox release 2014a.

[29] R. Warner, B. Rangan, A. Hall, K. Faulkes, Concrete structures, Addison Wesley Longman, 1998. 
[30] S. Wallah, B. V. Rangan, Low-calcium fly ash-based geopolymer concrete: long-term properties, Res. Report-GC2, Curtin University, Australia. 